\title{
A Hierarchical Recurrent Neuro-Fuzzy System
}

\author{
Andreas Nürnberger \\ University of Magdeburg, Faculty of Computer Science \\ 39106 Magdeburg, Germany \\ E-mail: andreas.nuernberger@cs.uni-magdeburg.de \\ Fax: +49 39167 12018, Phone: +49 3916711358
}

\begin{abstract}
Fuzzy systems, neural networks and its combination in neuro-fuzzy systems are already well established in data analysis and system control. Especially, neurofuzzy systems are well suited for the development of interactive data analysis tools, which enable the creation of rule-based knowledge from data and the introduction of a-priori knowledge into the process of data analysis. However, its recurrent variants - especially recurrent neuro-fuzzy models - are still rarely used. In this article a (hybrid) recurrent neuro-fuzzy model is presented which is designed for application in time series prediction and identification of dynamic systems. It has been implemented in a tool for the interactive design of hierarchical recurrent fuzzy systems.
\end{abstract}

\section{Introduction}

The main idea of neuro-fuzzy systems is to combine the advantages of fuzzy systems (e.g. interpretability, use of prior knowledge for initialization) with the learning capabilities of neural networks. Thus, using fuzzy rules, it is possible to interpret the network structure and to introduce prior knowledge to the learning process in a convenient way. A disadvantage of these approaches is that in most cases the quality of the solution result is reduced due to the constraints that ensure the interpretability. However, meanwhile a lot of models have been proposed for control [1, 2, 3, classification [4, 5] and function approximation [6, 7]. For a detailed overview of neuro-fuzzy systems see, for exampl@, [8, 20].

Despite of the research that has already been done in the area of neuro-fuzzy systems the recurrent variants of this architecture are still rarely studied. One of the first approaches was presented in 10 . However, in contrast to pure feed-forward architectures, that have a static input-output behavior, recurrent models are able to store information of the past (e.g. prior system states) and are thus more appropriate for the analysis of dynamic systems. If pure feed-forward architectures are applied to these types of problems (e.g. prediction of time series data or physical systems), the obtained system data usually has to be preprocessed or restructured to map the dynamic information appropriately. In this paper a model is presented that was designed to learn and optimize a hierarchical fuzzy rule base with feedback connections. The model is restricted to optimize fuzzy systems using Mamdani-like fuzzy rules. Logistic or Gaussian-like fuzzy sets can be used to define the membership functions of the antecedents and Gaussian-like fuzzy sets to define the consequents. In the following the structure of the model and the used learning methods are described. Furthermore, an application example is presented.

\section{Model Structure}

The main idea of this model is to combine simple feedforward fuzzy systems - which may consist of just one rule - to arbitrary hierarchical models. Therefore, the interpretability of every part is ensured before, during and after optimization. Backward connections between the models are realized by time-delayed feedback links. The interpretability of the fuzzy sets is ensured by the use of coupled weights in the consequents (fuzzy sets, which are assigned to the same linguistic terms share their parameters) and in the antecedents (layer two). Furthermore, constraints can be defined, which have to be observed by the learning method, e.g. that the fuzzy sets have to cover the considered state space. A possible structure is shown in Figure 1 A formal definition is given in the following.

Definition 1. Let Ant $(r)$ be the set of the fuzzy sets used in the antecedent and Con $(r)$ be the set of the fuzzy sets used in the consequent of rule $r$, then the recurrent neuro-fuzzy system is a fuzzy system with the following specifications:

(i) All fuzzy sets $\mu_{j}^{(i)}\left(x_{i}\right) \in \operatorname{Ant}(r)$ are defined by either Gaussian-like or logistic fuzzy sets

$$
\begin{gathered}
\mu_{j}^{(i)}\left(x_{i}\right)=e^{\frac{-\left(x_{i}-c_{j}^{(i)}\right)^{2}}{2\left(\alpha_{j}^{(i)}\right)^{2}}} \text { or } \\
\mu_{j}^{(i)}\left(x_{i}\right)=\frac{1}{1+e^{\beta_{j}^{(i)}\left(x_{i}-d_{j}^{(i)}\right)}},
\end{gathered}
$$


where $\mu_{j}^{(i)}$ is the $j$-th fuzzy set assigned to input $x_{i}$.

All fuzzy sets $v_{l}^{(k)}\left(y_{k}\right) \in \operatorname{Con}(r)$ are defined by Gaussian-like fuzzy sets

$$
v_{l}^{(k)}\left(y_{k}\right)=e^{\frac{-\left(y_{k}-\hat{c}_{c}^{(k)}\right)^{2}}{2\left(\hat{\alpha}_{l}^{(k)}\right)^{2}}},
$$

where $v_{l}^{(k)}$ is the l-th fuzzy set assigned to output $y_{k}$.

(ii) Each rule is a tuple

$$
R_{r}=\left(\mu_{j_{\eta}}^{\left(i_{\eta}\right)}, \ldots, \mu_{j_{r_{n}}}^{\left(i_{r_{n}}\right)}, v_{l_{\eta}}^{\left(k_{r_{n}}\right)}, \ldots, v_{l_{r_{n}}}^{\left(k_{r_{n}}\right)}\right)
$$

and has the form

$$
\begin{aligned}
& R_{r} \text { : if } x_{i_{r_{1}}} \text { is } \mu_{j_{r_{n}}}^{\left(i_{\eta^{\prime}}\right)} \text { and } \ldots \text { and } x_{i_{r_{n}}} \text { is } \mu_{j_{r_{n}}}^{\left(i_{r_{n}}\right)} \\
& \text { then } y_{k_{r_{n}}} \text { is } v_{l_{r_{n}}}^{\left(k_{r_{1}}\right)} \text { and } \ldots \text { and } y_{k_{r_{n}}} \text { is } v_{l_{r_{n}}}^{\left(k_{r_{n}}\right)} \text {. }
\end{aligned}
$$

(iii) The activation $a_{r}(t)$ of a fuzzy rule at time $t$ is computed by

$$
a_{r}(t)=\prod_{i, j: \mu_{j}^{(i)} \in \operatorname{Ant}(r)} \mu_{j}^{(i)}\left(x_{i}\right) .
$$

(iv) The output of the system for each output variable $y_{k}$ is computed by a weighted sum:

$$
\begin{aligned}
y_{k}(t) & =\frac{\sum_{r: l_{l_{r}}^{(k)} \in \operatorname{Con}(r)} \operatorname{area}\left(v_{l_{r}}^{(k)}, a_{r}(t)\right) \cdot \operatorname{center}\left(v_{l_{r}}^{(k)}, a_{r}(t)\right)}{\sum_{r: v_{l_{r}}^{(k)} \in \operatorname{Con}(r)} \operatorname{area}\left(v_{l_{r}}^{(k)}, a_{r}(t)\right)} \\
& =\frac{\sum_{r: l_{l_{r}}^{(k)} \in \operatorname{Con}(r)} \operatorname{area}\left(v_{l_{r}}^{(k)}, a_{r}(t)\right) \cdot \hat{c}_{l_{r}}^{(k)}}{\sum_{r: v_{l_{r}}^{(k)} \in \operatorname{Con}(r)} \operatorname{area}\left(v_{l_{r}}^{(k)}, a_{r}(t)\right)},
\end{aligned}
$$

where area computes the area and center the center of gravity of the output fuzzy set $\hat{v}_{l_{r}}^{(k)}$ of rule r for $y_{k}$ at time $t$ which is defined as

$$
\hat{v}_{l_{r}}^{(k)}\left(y_{k}\right)=\min \left(v_{l_{r}}^{(k)}\left(y_{k}\right), a_{r}(t)\right)
$$

(v) Two types of feedback connections are allowed:

a. Time-delayed feedback: An output $y_{k}$ can be assigned to an input $x_{i}$ by $x_{i}(t):=y_{k}(t-1)$.

b. Hierarchical feedback: An output $y_{k}$ can be assigned to an input $x_{i}$ by defining $x_{i}(t):=y_{k}(t)$ if $x_{i}(t)$ is not used for the computation of $y_{k}(t)$ neither directly nor indirectly via another ( $h$ ierarchical) input $x_{j}(t)$.

The feedback connections defined in (v) enable the definition of hierarchical fuzzy systems $(b$. ) and the use of time-delayed inputs (a.). However, only timedelayed feedback connections are considered as 'real' recurrent connections. Hierarchical feedback connec-

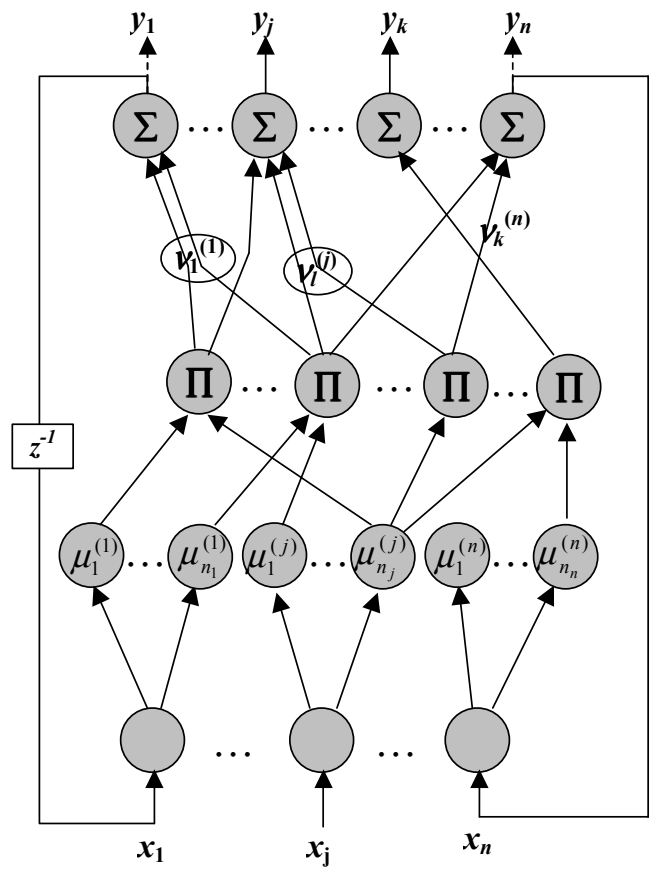

Figure 1. Possible structure of the proposed recurrent neuro-fuzzy system (using one time-delayed and one hierarchical feed-back)

tions are simply a 'recurrent' definition of a hierarchical fuzzy rule base. The condition defined in $b$. ensures that the rule base can be sorted topologically.

To simplify the definition of recurrent systems, a fuzzy set may be used in a rules consequent and antecedent. Therefore, also fuzzy sets $\mu_{j}^{(i)}$ and $v_{l}^{(k)}$ might share the same parameters. Nevertheless, a fuzzy set may be used at most once in a rules consequent and antecedent, respectively.

The used defuzzification method is a modified center of gravity approach (COG). The area below the Gaussian-like fuzzy sets is approximated - for performance reasons - by a triangle, where the center is defined as $\hat{c}_{l}^{(k)}$ and the left border $l_{l}^{(k)}$ and the right border $r_{l}^{(k)}$ are defined by $\hat{c}_{l}^{(k)} \mp 2.5 \cdot \hat{\alpha}_{l}^{(k)}$, respectively. Therefore, the area below $\hat{v}_{l}^{(k)}$ can be approximated by a trapezoid:

$$
\operatorname{area}\left(v_{l_{r}}^{(k)}, a_{r}(t)\right)=\left(r_{l_{r}}^{(k)}-l_{l_{r}}^{(k)}\right)\left(a_{r}(t)-\frac{1}{2} a_{r}(t)^{2}\right) .
$$

For the considered Gaussian-like fuzzy sets, this leads to

$$
\operatorname{area}\left(v_{l_{r}}^{(k)}, a_{r}(t)\right)=5 \cdot \alpha_{l_{r}}^{(k)}\left(a_{r}(t)-\frac{1}{2} a_{r}(t)^{2}\right) .
$$

\section{Learning Method}

The learning approach is separated in two learning phases: rule base learning and rule base optimization. The main idea of the rule base learning approach is to learn a hierarchically structured rule base of local sub- 
systems. This simplifies the introduction of prior knowledge and the interpretability of the complete rule base. To be able to learn subsystems, i.e. systems that use just a subset of input and output variables and probably additional inner variables, the algorithm supports the use of rule templates. These templates are used to define the variables and the belonging time delays that should be used for the creation of a specific subsystems. Thus, it is possible to optimize each subsystem independently of another, but it is also ensured that each subsystem, and thus the complete rule base, is still interpretable. The learning method used for each subsystem is motivated by the heuristics used in the NEFPROX model [7]. The algorithm requires an existing fuzzy partitioning for each considered domain.

The proposed method for rule base optimization is motivated by the real-time recurrent learning method (RTRL) for recurrent neural networks [11]. The idea is to propagate the error obtained at the output units back through the rules of the (hierarchical) fuzzy system and adapt the fuzzy sets accordingly. If feed-back connections have to be considered, the fuzzy rule base is unfolded in time and the error is propagated back through time. A detailed description of the algorithm is given in the following.

Let $\mathrm{E}$ be the total error (cost function to be minimized) of all output neurons k over all time steps $t=0, \ldots, T$ :

$$
\begin{gathered}
E=\sum_{t=0}^{T} E(t), \text { with } \\
E(t)=\frac{1}{2} \sum_{k}\left(E_{k}(t)\right)^{2}, \text { and } \\
E_{k}(t)= \begin{cases}o_{k}(t)-y_{k}(t) & \text { if node } \mathrm{k} \text { has a target } \\
0 & \text { output } o_{k} \text { at time } t,\end{cases}
\end{gathered}
$$

be an error measure for output node $\mathrm{k}$. Then the error gradient of error $E(t)$ for an arbitrary parameter $p$ of the considered fuzzy rule base is

$$
\frac{\partial E(t)}{\partial p}=-\sum_{k} E_{k}(t) \frac{\partial y_{k}(t)}{\partial p} .
$$

Since the activation of a rule $a_{r}(t)$ may depend on parameter $p$, the partial derivatives with respect to $a_{r}(t)$ have to be considered. (For formal reasons a parameter of the fuzzy system is represented in the following by a function $f$, with $f_{p}(p):=p$.) This leads to

$$
\begin{aligned}
\frac{\partial y_{k}(t)}{\partial p} & =\frac{\partial y_{k}(t)}{\partial f_{p}(p)} \frac{\partial f(p)}{\partial p}+\sum_{r: v_{l_{r}}^{(k)} \in \operatorname{Con}(r)} \frac{\partial y_{k}(t)}{\partial a_{r}(t)} \frac{\partial a_{r}(t)}{\partial p} \\
& =\frac{\partial y_{k}(t)}{\partial f_{p}(p)}+\sum_{r: v_{l_{r}}^{(k)} \in \operatorname{Con}(r)} \frac{\partial y_{k}(t)}{\partial a_{r}(t)} \frac{\partial a_{r}(t)}{\partial p} .
\end{aligned}
$$

Furthermore, the derivatives of the input $x_{i}(t)$ with respect to $p$ have to be considered:

$$
\begin{aligned}
\frac{\partial a_{r}(t)}{\partial p} & =\frac{\partial a_{r}(t)}{\partial f_{p}(p)} \frac{\partial f(p)}{\partial p}+\sum_{i: \mu_{j_{r}}^{(i)} \in A n t(r)} \frac{\partial a_{r}(t)}{\partial x_{i}(t)} \frac{\partial x_{i}(t)}{\partial p} \\
& =\frac{\partial a_{r}(t)}{\partial f_{p}(p)}+\sum_{\substack{i: \mu_{j_{r}}^{(i)} \in \operatorname{Ant}(r) \\
\frac{\partial a_{r}(t)}{\partial x_{i}(t)}}}^{\frac{\partial x_{i}(t)}{\partial p} .}
\end{aligned}
$$

Therefore, we finally obtain

$$
\begin{aligned}
\frac{\partial y_{k}(t)}{\partial p} & =\frac{\partial y_{k}(t)}{\partial f_{p}(p)}+ \\
\sum_{r: v_{l_{r}}^{(k)} \in \operatorname{Con}(r)} & {\left[\frac{\partial y_{k}(t)}{\partial a_{r}(t)} \cdot\left(\frac{\partial a_{r}(t)}{\partial f_{p}(p)}+\sum_{i: \mu_{j r}^{(i)} \in A n t(r)} \frac{\partial a_{r}(t)}{\partial x_{i}(t)} \frac{\partial x_{i}(t)}{\partial p}\right)\right] }
\end{aligned}
$$

Now, the specific derivatives have to be determined. For $\frac{\partial x_{i}(t)}{\partial p}$ four cases have to be considered:

$$
\frac{\partial x_{i}(t)}{\partial p}= \begin{cases}\frac{\partial y_{j}(t)}{\partial p} & \text { If } \frac{\partial x_{i}(t)}{\partial p} \text { is a hierarchical } \\ \text { feedback from } y_{j}(t), \\ \frac{\partial y_{j}(t-1)}{\partial p} & \text { If } \frac{\partial x_{i}(t)}{\partial p} \text { is a time delayed } \\ \frac{\partial x_{j}(t-1)}{\partial p} & \text { feedback from } y_{j}(t-1) \\ 0 & \text { If } \frac{\partial x_{i}(t)}{\partial p} \text { is a self - feedback }\end{cases}
$$

If $x_{i}(t)$ is an external input to the system, then $\frac{x_{i}(t)}{\partial p}=0$ since the external input is independent from any parameter of the system (neglecting any external recurrences, which could not be handled by the system itself).

If $x_{i}(t)$ is assigned to an internal variable of the system, three cases have to be considered. For a hierarchical feedback with $x_{i}(t):=y_{i}(t), \frac{x_{i}(t)}{\partial p}$ can be computed by (3.7) by replacing $x_{i}(t)$ with the output variable $y_{j}(t)$ of the preceding layer, since a hierarchical feedback is independent from $x_{i}$. If $x_{i}(t)$ is a selffeedback input, i.e. $x_{i}(t):=x_{j}(t-1)$, then $\frac{x_{i}(t)}{\partial p}$ can be computed iteratively similar to RTRL learning, by assuming $\frac{\partial x_{i}(0)}{\partial p}=0$, since the initial state can be considered as independent from $p$. If $x_{i}(t)$ is a time-delayed feedback input, i.e. $x_{i}(t):=y_{j}(t-1)$, then 
$\frac{\partial x_{i}(t)}{\partial p}=\frac{\partial y_{j}(t-1)}{\partial p}$ and $\frac{\partial y_{j}(t)}{\partial p}$ can be computed by To simplify the derivation of the remaining derivatives, let

$$
\begin{aligned}
A^{(k)}(t) & :=\sum_{j: v_{l_{j}}^{(k)} \in \operatorname{Con}(j)} \operatorname{area}\left(v_{l_{j}}^{(k)}, a_{j}(t)\right) \cdot \hat{c}_{l_{j}}^{(k)}, \\
B^{(k)}(t) & :=\sum_{j::_{l_{j}}^{(k)} \in \operatorname{Con}(j)} \operatorname{area}\left(v_{l_{j}}^{(k)}, a_{j}(t)\right) .
\end{aligned}
$$

We obtain

$$
\begin{aligned}
& \frac{\partial y_{k}(t)}{\partial a_{r}(t)}=\frac{\partial}{\partial a_{r}(t)}\left(\frac{A^{(k)}(t)}{B^{(k)}(t)}\right) \\
& =\frac{\partial}{\partial a_{r}(t)}\left(\operatorname{area}\left(v_{r}^{(k)}, a_{r}(t)\right)\right) \cdot \frac{\left(\hat{c}_{l_{r}}^{(k)} B^{(k)}(t)-A^{(k)}(t)\right)}{\left(B^{(k)}(t)\right)^{2}} \\
& =5 \cdot \hat{\alpha}_{l_{r}}^{(k)}\left(1-a_{r}(t)\right) \cdot \frac{\left(\hat{c}_{l_{r}}^{(k)} B^{(k)}(t)-A^{(k)}(t)\right)}{\left(B^{(k)}(t)\right)^{2}}
\end{aligned}
$$

where $\hat{c}_{l_{r}}^{(k)}$ is the parameter of the Gaussian-like fuzzy set assigned to the consequent of rule $r$ for output $y_{k}$. For $\frac{\partial a_{r}(t)}{\partial x_{i}(t)}$ we obtain

$$
\begin{aligned}
\frac{\partial a_{r}(t)}{\partial x_{i}(t)} & =\frac{\partial}{\partial x_{i}(t)}\left(\prod_{l, m: \mu_{m}^{(l)} \in \operatorname{Ant}(r)} \mu_{m}^{(l)}\left(x_{l}\right)\right) \\
& =\frac{\partial}{\partial x_{i}(t)} \mu_{m_{r}}^{(i)}\left(x_{i}(t)\right) \cdot \prod_{\substack{l, m: \mu_{m}^{(l)} \in \text { Ant }(r) \\
l \neq i}} \mu_{m}^{(l)}\left(x_{l}(t)\right),
\end{aligned}
$$

where $\frac{\partial}{\partial x_{i}(t)} \mu_{m_{r}}^{(i)}\left(x_{i}(t)\right)$ depends on the type of the membership function $\mu_{m_{r}}^{(i)}(x)$. For Gaussian-like functions this leads to

$$
\frac{\partial}{\partial x_{i}(t)} \mu_{m_{r}}^{(i)}\left(x_{i}(t)\right)=\frac{-\left(x_{i}(t)-c_{m_{r}}^{(i)}\right)}{\left(\alpha_{m_{r}}^{(i)}\right)^{2}} \mu_{m_{r}}^{(i)}\left(x_{i}(t)\right),
$$

and for logistic functions we obtain

$$
\begin{aligned}
& \frac{\partial}{\partial x_{i}(t)} \mu_{m_{r}}^{(i)}\left(x_{i}(t)\right)= \\
& \quad-\beta_{m_{r}}^{(i)} \cdot e^{\beta_{m_{r}}^{(i)}\left(x_{i}(t)-d_{m_{r}}^{(i)}\right)} \cdot\left(\mu_{m_{r}}^{(i)}\left(x_{i}(t)\right)\right)^{2} .
\end{aligned}
$$

The remaining derivatives with respect to the considered parameter $f_{p}(p)$ are given in the following.

\subsection{Derivations for Consequent Parameters}

For the adaptation of the parameters $\hat{c}_{l}^{(k)}$ and $\hat{\alpha}_{l}^{(k)}$ of the Gaussian-like fuzzy sets used in the consequent of rule $r$, the parameter defined by $f_{p}(p)$ in Equation (3.7) has to be replaced by $\hat{c}_{l}^{(k)}$ and $\hat{\alpha}_{l}^{(k)}$, respectively.
Therefore, we obtain for $f_{p}(p)=\hat{c}_{i}^{(k)}$ (the indirect dependencies of $a_{r}(t)$ and $x_{i}(t)$ on the parameters $\hat{c}_{l}^{(k)}$ and $\hat{\alpha}_{l}^{(k)}$ are already taken into account by the application of the chain rule in (3.7) Here, only the derivation with respect to explicit occurrences of the parameters have to be derived)

$$
\frac{\partial a_{r}(t)}{\partial f_{p}(p)}=\frac{\delta a_{r}(t)}{\delta \hat{c}_{l}^{(k)}}=0,
$$

since $a_{r}(t)$ does not directly depend on a parameter of the consequent, and

$$
\begin{aligned}
\frac{\partial y_{k}(t)}{\partial f_{p}(p)} & =\frac{\delta y_{k}(t)}{\delta \hat{c}_{l}^{(k)}} \\
& =\frac{\delta}{\delta \hat{c}_{l}^{(k)}}\left(\frac{\sum_{r: l_{l_{r}}^{(k)} \in \operatorname{Con}(r)} \operatorname{area}\left(v_{l_{r}}^{(k)}, a_{r}(t)\right) \cdot \hat{c}_{l_{r}}^{(k)}}{\sum_{r: v_{l_{r}}^{(k)} \in \operatorname{Con}(r)} \operatorname{area}\left(v_{l_{r}}^{(k)}, a_{r}(t)\right)}\right) \\
& =\frac{\sum_{j: l_{l_{j}}^{(k)} \in \operatorname{Con}(j) \wedge l_{j}=l} \operatorname{area}\left(v_{l}^{(k)}, a_{j}(t)\right)}{\sum_{r: v_{l_{r}}^{(k)} \in \operatorname{Con}(r)} \operatorname{area}\left(v_{l_{r}}^{(k)}, a_{r}(t)\right)} .
\end{aligned}
$$

Analogously, for $f_{p}(p)=\hat{\alpha}_{l}^{(k)}$ we obtain

$$
\frac{\partial a_{r}(t)}{\partial f_{p}(p)}=\frac{\delta a_{r}(t)}{\delta \hat{\alpha}_{i}^{(k)}}=0
$$

and

$$
\begin{aligned}
& \frac{\partial y_{k}(t)}{\partial f_{p}(p)}=\frac{\delta y_{k}(t)}{\delta \hat{\alpha}_{l}^{(k)}}=\frac{\delta}{\delta \hat{\alpha}_{l}^{(k)}}\left(\frac{A^{(k)}(t)}{B^{(k)}(t)}\right) \\
& =\frac{\left(\hat{c}_{l}^{(k)} B^{(k)}(t)-A^{(k)}(t)\right)}{\left(B^{(k)}(t)\right)^{2}} \\
& \cdot \sum_{j: l_{l_{j}}^{(k)} \in \operatorname{Con}(j) \wedge l_{j}=l}\left[\frac{\delta}{\delta \hat{\alpha}_{l}^{(k)}}\left(\operatorname{area}\left(v_{l}^{(k)}, a_{j}(t)\right)\right)\right] \\
& =\frac{\left(\hat{c}_{l}^{(k)} B^{(k)}(t)-A^{(k)}(t)\right)}{\left(B^{(k)}(t)\right)^{2}} \\
& \sum_{j: v_{l j}^{(k)} \in \operatorname{Con}(j) \wedge l_{j}=l}\left[5 \cdot\left(a_{j}(t)-\frac{1}{2} a_{j}(t)^{2}\right)\right] \text {. }
\end{aligned}
$$

\subsection{Derivations for Antecedent Parameters}

Analogously to the derivation of the consequent parameters, for the parameters $c_{j}^{(i)}$ and $\alpha_{j}^{(i)}$ of a Gaussian-like fuzzy set, and $d_{j}^{(i)}$ and $\beta_{j}^{(i)}$ of a logistic fuzzy set used in the antecedent of rule $r$ the following derivatives are obtained $\left(y_{k}(t)\right.$ and $x_{i}(t)$ can, analogous to $a_{r}(t)$ and $x_{i}(t)$ in the previous section, be considered as independent from the parameters, since the indirect dependencies have already been taken into 
account in (3.7). Let $p$ be one of the considered parameters $c_{j}^{(i)}, \alpha_{j}^{(i)}, d_{j}^{(i)}$ or $\beta_{j}^{(i)}$, then we obtain

$$
\frac{\partial y_{k}(t)}{\partial f_{p}(p)}=0
$$

For $\frac{\partial a_{r}(t)}{\partial f_{p}(p)}$ we have (since $p$ represents a parameter of the $j$-th fuzzy set assigned to input $x_{i}$ )

$$
\begin{gathered}
\frac{\partial a_{r}(t)}{\partial f_{p}(p)}= \\
\left\{\frac{\partial}{\partial f_{p}(p)}\left(\prod_{s, t: \mu_{t_{r}}^{(s)} \in \operatorname{Ant}(r)} \mu_{t_{r}}^{(s)}\left(x_{s}(t)\right)\right)\right. \\
0 \quad \text { if } \mu_{j}^{(i)} \in \operatorname{Ant}(r), \\
\text { otherwise, }
\end{gathered}
$$

where

$$
\begin{aligned}
& \frac{\partial}{\partial f_{p}(p)}\left(\prod_{s, t: \mu_{t_{r}}^{(s)} \in \operatorname{Ant}(r)} \mu_{t_{r}}^{(s)}\left(x_{s}(t)\right)\right)= \\
& \frac{\partial}{\partial f_{p}(p)} \mu_{j}^{(i)}\left(x_{i}(t)\right) \cdot \prod_{\substack{s, t: \mu_{t r}^{(s)} \in \operatorname{Ant}(r) \\
s \neq i \wedge t \neq j}} \mu_{t_{r}}^{(s)}\left(x_{s}(t)\right) .
\end{aligned}
$$

By replacing $f_{p}(p)$ with the specific parameters we finally obtain the derivations of $\mu_{j}^{(i)}\left(x_{i}(t)\right)$ with respect to the parameters $c_{j}^{(i)}$ and $\alpha_{j}^{(i)}$ if $\mu_{j}^{(i)}$ defines a Gaussian-like fuzzy set, and $d_{j}^{(i)}$ and $\beta_{j}^{(i)}$ if $\mu_{j}^{(i)}$ defines a logistic fuzzy set. The derivations are given in the following.

$$
\begin{aligned}
\frac{\delta}{\delta c_{j}^{(i)}} \mu_{j}^{(i)}\left(x_{i}(t)\right) & =\frac{\left(x_{i}(t)-c_{j}^{(i)}\right)}{\left(\alpha_{j}^{(i)}\right)^{2}} \mu_{j}^{(i)}\left(x_{i}(t)\right), \\
\frac{\delta}{\delta \alpha_{j}^{(i)}} \mu_{j}^{(i)}\left(x_{i}(t)\right) & =\frac{\left(x_{i}(t)-c_{j}^{(i)}\right)^{2}}{\left(\alpha_{j}^{(i)}\right)^{3}} \mu_{j}^{(i)}\left(x_{i}(t)\right), \\
\frac{\delta}{\delta d_{j}^{(i)}} \mu_{j}^{(i)}\left(x_{i}(t)\right) & =\beta_{j}^{(i)} \cdot e^{\beta_{j}^{(i)}\left(x_{i}(t)-d_{j}^{(i)}\right)} \cdot\left(\mu_{j}^{(i)}\left(x_{i}(t)\right)\right)^{2}, \\
\frac{\delta}{\delta \beta_{j}^{(i)}} \mu_{j}^{(i)}\left(x_{i}(t)\right) & =-\left(x_{i}(t)-d_{j}^{(i)}\right) \cdot e^{\beta_{j}^{(i)}\left(x_{i}(t)-d_{j}^{(i)}\right)} \\
& \cdot\left(\mu_{j}^{(i)}\left(x_{i}(t)\right)\right)^{2} .
\end{aligned}
$$

Based on Equations (3.4) and (3.7) we can now compute the error gradients for the parameters of the fuzzy sets.

\section{Application Example}

In the simple example presented in the following we try to identify a spring-mass-model, which can be defined by the differential equation

$$
\ddot{x}=\frac{-c}{m} x,
$$

with the initial values $x(0)=x_{0}$ and $v(0)=\dot{x}(0)=0$.
This second order differential equation can be transformed into a system of two coupled first order differential equations by introducing an intermediary variable $v$

$$
\dot{x}=v \text { and } \dot{v}=\frac{-c}{m} x \text {. }
$$

As system parameters the values $c=40, m=1$ and $x_{0}=1$ were used. A data set was generated by simulating the system for a period of $20 \mathrm{sec}$. with a (fixed step size $\Delta t=0.1)$ explicit fourth-order Runge-Kutta method and storing the values $x(t)$ and $v(t)$. To obtain a rule base that can be interpreted with respect to each variable, two rule templates - one for the computation of $x$ and one for the computation of $v$ - were defined for the rule base learning method. The domains $x$ and $v$ were partitioned by three Gaussian-like fuzzy sets (neg, zero, pos), while for each subsystem and delay independent fuzzy sets were defined with similar initial parameters. The use of independent fuzzy sets for output and (time-delayed) input is necessary to allow each system to scale between the output and input values. The rule base learning method yielded the following rules:

IF ( $x[t-1]$ IS neg2 AND v[t-1] IS neg1) THEN $x$ IS neg0 IF (x[t-1] IS neg2 AND v[t-1] IS zero1) THEN $x$ IS neg0 IF ( $x[t-1]$ IS neg2 AND v[t-1] IS pos1) THEN $x$ IS zero0 IF (x[t-1] IS zero2 AND v[t-1] IS neg1) THEN $x$ IS neg0

IF ( $x[t-1]$ IS zero2 AND v[t-1] IS zero1) THEN $x$ IS zero0 IF $(x[t-1]$ IS zero2 AND $v[t-1]$ IS pos 1$)$ THEN $x$ IS pos0 IF (x[t-1] IS pos2 AND v[t-1] IS neg1) THEN $x$ IS zero0 IF (x[t-1] IS pos2 AND v[t-1] IS zero1) THEN $x$ IS pos0 IF (x[t-1] IS pos2 AND v[t-1] IS pos1) THEN $x$ IS pos 0

IF ( $x[t-1]$ IS neg1 AND v[t-1] IS neg2) THEN v IS zero0 IF ( $x[\mathrm{t}-1]$ IS neg1 AND $v$ [t-1] IS zero2) THEN $v$ IS pos0 IF (x[t-1] IS neg1 AND v[t-1] IS pos2) THEN $v$ IS pos0 IF (x[t-1] IS zero1 AND v[t-1] IS neg2) THEN $v$ IS neg0 IF ( $x[\mathrm{t}-1]$ IS zero1 AND v[t-1] IS zero2) THEN $v$ IS zero0 IF ( $x[\mathrm{t}-1]$ IS zero1 AND v[t-1] IS pos2) THEN $v$ IS pos0 IF ( $x[t-1]$ IS pos1 AND v[t-1] IS neg2) THEN $v$ IS neg0 IF ( $x[t-1]$ IS pos1 AND v[t-1] IS zero2) THEN $v$ IS neg0 IF ( $x[t-1]$ IS pos1 AND v[t-1] IS pos2) THEN $v$ IS zero0

The learned rule base of the subsystems for the computation of $x$ and $v$ are similar to the rule base of a simple integrator (adder). So, we can suppose, that $x$ is computed by integration of $v$, and $v$ by integration of (negated) $x$. A comparison to the formal definition proved this assumption (Equation (4.1).

After rule base learning, the rule base was optimized for 4000 training cycles (complete propagations through time) using $x$ and $v$ as training data. Figure 2 depicts the learning progress. The learning terminated with a summed square error of $E=12.872$, which is caused by the deviation from the frequency and amplitude of the physical system. In Figure 3 the position $x$ and velocity $v$ of the trained system and the validation data over time is shown ( $x$ and $v$ given). To evaluate the performance of the presented approach for inner 
variables, in following runs either $x$ or $v$ was presented to the system for learning, thus the other variable was considered as unknown. The simulation run using just $x$ for training yielded the best approximation of the amplitudes for $x$ and $v$, while the system which was trained by use of $v$ fits the frequencies very well (see Figure 3.

It has to be emphasized, that the only input to the system are the initial values $x(0)=1$ and $v(0)=0$. The system used its own output as input and just the error signal $E_{i}(t)$ - the difference between the system output and training data - for learning.

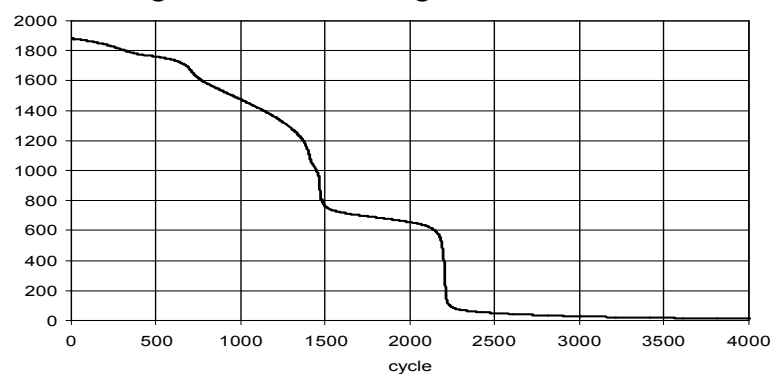

Figure 2. Change of $E(t)$ ( $x$ and $v$ given)
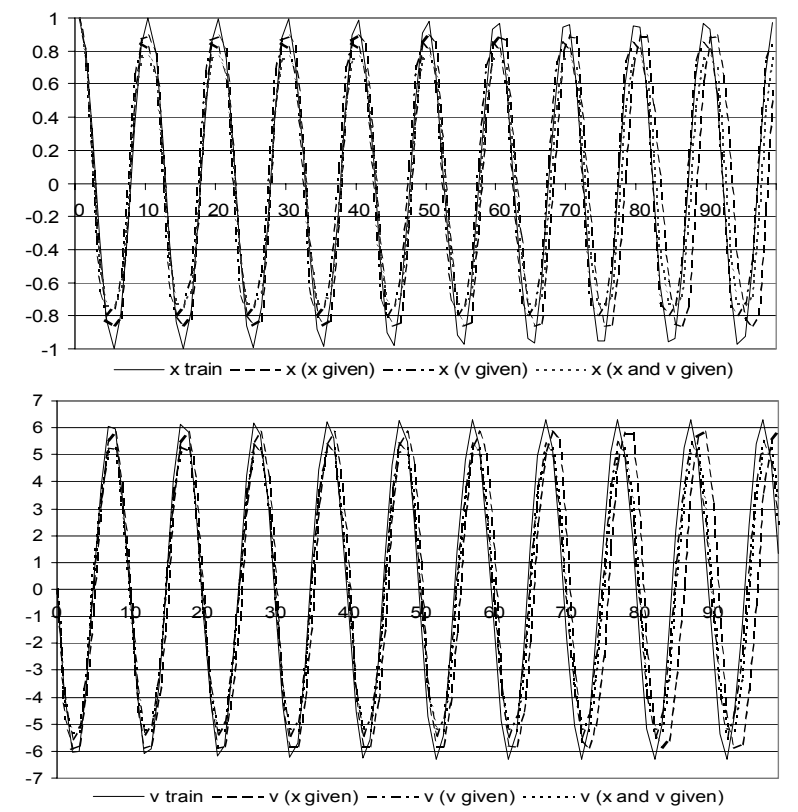

Figure 3. System output

\section{Conclusions}

As presented, the proposed model can be used to learn and optimize recurrent fuzzy rule bases using rule templates. Furthermore, the rule base optimization method can be used to optimize arbitrary hierarchical recurrent rule bases.

Unfortunately, recurrent systems are usually more sensitive to small changes in the variables, since the (error prone) output of the system is also used as feedback input and therefore the error might reinforce itself. For that reason, it is usually more important to obtain a good approximation performance, than to ensure a good interpretability as it is usually the goal in the design of feed-forward neuro-fuzzy approaches. The learning constraints, which are used in feed-forward architectures to ensure a good interpretability of the learned fuzzy rule base, usually have to be relaxed or even neglected to obtain a working solution. However, as it is shown in the presented examples, it is still possible to interpret the obtained rule base and thus to extract knowledge about the analyzed system.

\section{References}

[1] H. R. Berenji, and P. Khedkar, "Learning and Tuning Fuzzy Logic Controllers through Reinforcements," IEEE Trans. Neural Networks, 3, 1992, pp 724-740.

[2] D. Nauck, F. Klawonn, and R. Kruse, Foundations of Neuro-Fuzzy Systems, Wiley, Chichester, 1997.

[3] A. Nürnberger, D. Nauck, and R. Kruse, "NeuroFuzzy Control Based on the NEFCON-Model," Soft Computing, 2(4), Springer, Berlin, 1999, pp 182-186.

[4] N. Tschichold-Gürman, "The Neural Network Model RuleNet and its Application to Mobile Robot Navigation," Fuzzy Sets and Systems, 85, 1997, pp 287-303.

[5] D. Nauck, "Knowledge Discovery with NEFCLASS," Proc. Fourth Int. Conf. Knowledge Based Intell. Eng. Systems \& Allied Technologies (KES'2000), Brighton, 2000, pp 158-161.

[6] J.-S. R. Jang, “ANFIS: Adaptive-Network-Based Fuzzy Inference Systems," IEEE Trans. Systems, Man \& Cybernetics, 23, 1993, pp 665-685.

[7] D. Nauck, and R. Kruse, "Function Approximation by NEFPROX," Proc. Sec. European Workshop on Fuzzy Decision Anal. and Neural Networks for Management, Planning, and Optimization (EFDAN'97), Dortmund, 1997, pp 160-169.

[8] C.-T. Lin, and C.-C. Lee, Neural Fuzzy Systems. A Neuro-Fuzzy Synergism to Intelligent Systems, Prentice Hall, New York, 1996.

[9] W. Pedrycz, Computational Intelligence: An Introduction, CRC Press., Boca Raton, FL, 1998.

[10] V. Gorrini, and H. Bersini, "Recurrent Fuzzy Systems," Proc. of the 3rd Conference on Fuzzy Systems (FUZZ-IEEE 94), IEEE, Orlando, 1994.

[11] R. J. Williams, and D. Zipser, "Experimental analysis of the real time recurrent learning algorithm," Connection Science, 1, 1989, pp 87-111. 